\title{
Constant False Alarm Rate Sound Source Detection with Distributed Microphones
}

\author{
Kevin D. Donohue, Sayed M. SaghaianNejadEsfahani, and Jingjing Yu \\ Department of Electrical and Computer Engineering, University of Kentucky, Lexington, KY 40506, USA \\ Correspondence should be addressed to Kevin D. Donohue, donohue@engr.uky.edu
}

Received 5 March 2010; Accepted 24 January 2011

Academic Editor: Sven Nordholm

Copyright () 2011 Kevin D. Donohue et al. This is an open access article distributed under the Creative Commons Attribution License, which permits unrestricted use, distribution, and reproduction in any medium, provided the original work is properly cited.

\begin{abstract}
Applications related to distributed microphone systems are typically initiated with sound source detection. This paper introduces a novel method for the automatic detection of sound sources in images created with steered response power (SRP) algorithms. The method exploits the near-symmetric coherent power noise distribution to estimate constant false-alarm rate (CFAR) thresholds. Analyses show that low-frequency source components degrade CFAR threshold performance due to increased nonsymmetry in the coherent power distribution. This degradation, however, can be offset by partial whitening or increasing differential path distances between the microphone pairs and the spatial locations of interest. Experimental recordings are used to assess CFAR performance subject to variations in source frequency content and partial whitening. Results for linear, perimeter, and planar microphone geometries demonstrate that experimental false-alarm probabilities for CFAR thresholds ranging from $10^{-1}$ and $10^{-6}$ are limited to within one order of magnitude when proper filtering, partial whitening, and noise model parameters are applied.
\end{abstract}

\section{Introduction}

Automatic sound source detection with distributed microphone systems is relevant for enhancing applications such as teleconferencing $[1,2]$, speech recognition [3-6], talker tracking [7], and beamforming [8]. Many of these applications involve the detection and location of sound sources. For example, an automatic minute-taking application must detect and locate active voices before beamforming to create independent channels for each speaker. Failure to detect active sound sources or false detections will degrade performance. This paper, therefore, introduces a method for automatically detecting sound sources using a variant of the steered response power (SRP) algorithm and applying a novel constant false-alarm rate (CFAR) threshold algorithm.

Recent work has shown the SRP algorithm to be robust in reverberant and multiple speaker environments when used in conjunction with a phase transform (PHAT) [9, 10]. The PHAT whitens the signals by setting the Fourier magnitudes to unity while maintaining the original phase. A detailed analysis based on detection performance showed that a variant of the PHAT, referred to as partial whitening or PHAT- $\beta[11,12]$, outperforms the PHAT for a variety of signal source types typically found in speech. Detection performance was analyzed using receiver operating characteristic (ROC) curve areas, which reflect overall detection and false-alarm performance without regard to a threshold.

A CFAR threshold is typically estimated based on a probabilistic model of the noise-only distribution, such that parameters are estimated from the local data to maintain a fixed probability of false alarm over nonstationarities. Adaptive thresholding algorithms based on a CFAR approach are common in radar and other applications, where large amounts of nonstationary noise samples are available [1315]. The CFAR algorithm presented here differs from previous approaches in that it uses coherent power. The coherent power is the sum of correlations between signals from all distinct microphone pairs focused on a point of interest (where no microphone signal is correlated with itself). This can be computed by subtracting the power of each individual microphone signal from the usual SRP value to create an acoustic image with positive and negative values. While common CFAR approaches use the cells or pixels (which are all positive) in the test pixel neighborhood to estimate 
the FA threshold, the approach described in this paper distinguishes itself by exploiting a distribution similarity between the positive and negative coherent noise pixels. The CFAR threshold is computed only from the absolute values of the negative pixels in the test pixel neighborhood. The omission of positive values in the threshold estimation results in a more consistent false-alarm rate, since (as will be seen in Section 4) the negative coherent power values are not as sensitive to the partial coherences from interfering sources. In addition, when a target is present and skews the positive neighboring pixels, the positive values do not bias the threshold high and lower detection sensitivity.

This approach was motivated by the observation that noise-only regions of coherent power pixels tend to be symmetrically distributed about zero over local neighborhoods, while for target regions the distributions were highly skewed in the positive direction. This observation was first exploited in [16], which demonstrated the CFAR method with limited data and analyses. The work in this paper establishes the relationship between the symmetry of the coherent power distribution and sensor placement in relationship to the field of view (FOV), as well as signal processing methods useful for improving CFAR performance. A characterization for microphone and FOV geometries is presented based on the interpath difference distributions of microphone pairs to FOV points. It is shown that when this distribution has a small variance relative to the source wavelengths, the distribution of the coherent power pixels lacks symmetry, which limits application of CFAR threshold method presented here. The small interpath distribution is typically the case for many far-field applications in radar and sonar, which is likely a reason why the idea of using negative-only coherent power values did not immerge in their CFAR literature. The symmetric distribution, however, occurs more naturally for immersive applications where the microphones surround the FOV. The analyses in this paper consider 3 array geometries to illustrate this effect relative to CFAR performance.

The issues related to good performance with this approach include determining the factors that impact the coherent power symmetry and finding statistical characterizations between the negative and positive coherent power values that lead to accurate threshold estimation. Therefore, this paper presents statistical analyses of coherent power values to assess noise modeling and signal processing approaches for enhancing CFAR performance. The analysis in this work shows analytically and experimentally that the primary source of performance degradation is the inability of a given microphone distribution to decorrelate lowfrequency components. Statistics based on the microphone geometry and FOV are derived to assess the ability of the microphone distribution in combination with signal processing techniques to yield near-symmetric noise distributions. Results show how signal processing techniques can be applied to reduce degradation from low frequencies.

This paper is organized as follows. Section 2 presents equations for creating an acoustic image based on the steered-response coherent power (SRCP) algorithm and derives statistics related to the noise distribution symmetry. Section 3 describes the microphone distributions and FOV geometries used in the experiments. Frequency ranges for each array are derived for achieving sufficient distribution symmetry. Section 4 directly analyzes the noise distributions with the Weibull distribution for various frequency limits and degrees of partial whitening. Section 5 presents the CFAR algorithm and performance analyses using data recorded from the three different microphone distributions and discusses the results. Finally, Section 6 summarizes the results and presents conclusions.

\section{Noise Distribution Factors}

2.1. Steered Response Coherent Power Images. This section derives the SRP algorithm for creating acoustic images in terms of coherent power rather than power. The use of coherent power is critical for this CFAR threshold algorithm because only pixels with negative values in the test pixel neighborhood are used to compute the threshold for the positive pixels. While derivations show that perfect symmetry cannot be expected, the factors influencing the deviations from symmetry are identified, so signal processing or array modifications can be applied to reduce these deviations and achieve good CFAR performance. The noise model considered in this derivation does not include electronic noise or contributions from continuously distributed sources. These noise sources do not significantly impact the symmetry in coherent power distributions. Point sources, on the other hand, create partial coherences throughout the FOV (due to beamformer sidelobes) and more directly impact the performance of this technique (as well as other SPR methods). Therefore, to simplify the notation and focus on aspects more critical to the performance, the noise model is limited to point sources not at the position being tested.

The following derivation expands a similar derivation presented in [16] to include the partial whitening operation and exclusively considers test positions in the FOV that contain no sources. The noise is modeled as a discrete spatial distribution of point sources located away from the test position. Consider a distribution of $P$ microphones, where vector $\mathbf{r}_{p}$ denotes the position of the $p$ th microphone. The waveform received by the $p$ th microphone can be written as

$$
u_{p}\left(t ; \mathbf{r}_{p}\right)=\sum_{k=1}^{K} \int_{-\infty}^{\infty} h_{k p}(\lambda) n_{k}(t-\lambda) d \lambda,
$$

where $n_{k}(t)$ represents noise source located at $\mathbf{r}_{k}, K$ is the number of effective noise sources contributing the $p$ th microphone signal, and $h_{k p}(\cdot)$ represents the impulse response for the room (including multipath) for the path from $\mathbf{r}_{k}$ to $\mathbf{r}_{p}$.

An SRP pixel value is based on sound events contributing to the signal over a finite time frame denoted by $\Delta_{l}$. A frame for a single channel in frequency domain is given by

$$
\hat{U}_{p}\left(\omega, \Delta_{l}\right)=\sum_{k \in 1}^{K} \hat{N}_{k}(\omega) \hat{A}_{k p}(\omega) \exp \left(-j \omega \tau_{k p}\right),
$$

where $\hat{N}_{k}(\omega)$ is the Fourier transform of the noise source signal over $\Delta_{l}, \hat{A}_{k p}(\omega)$ is the noise source path transfer 
function to the $p$ th microphone with the time delay, $\tau_{k p}$, factored out, and the summation is only over the $K$ effective sources with path delays falling within interval $\Delta_{l}$.

At this point, whitening can be applied to each microphone signal via the PHAT- $\beta$ denoted by

$$
\hat{V}_{p}(\omega, l)=\frac{\hat{U}_{p}\left(\omega, \Delta_{l}\right)}{\left|\hat{U}_{p}\left(\omega, \Delta_{l}\right)\right|^{\beta}},
$$

where $\beta$ can be chosen on the interval [ $\left[\begin{array}{ll}0 & 1\end{array}\right]$ to achieve various degrees of whitening, where $\beta$ equal to zero results in no whitening, and $\beta$ equal to 1 results in total whitening as in the PHAT $[9,10]$. Other values of $\beta$ result in partial whitening as in the case of the PHAT- $\beta[11,12]$.

The SRP pixel value, corresponding to $\mathbf{r}_{i}$, is computed from the signal power at the $l$ th time frame

$$
S\left(\mathbf{r}_{i}, l\right)=\int_{\omega} \mathbf{B}_{i} \mathbf{V}(\omega, l) \mathbf{V}^{H}(\omega, l) \mathbf{B}_{i}^{H} d \omega,
$$

where superscript $H$ denotes the complex conjugate transpose. $\mathbf{B}_{i}$ is the steering vector of the form

$$
\mathbf{B}_{i}=\left[\widehat{B}_{i 1}, \hat{B}_{i 2}, \ldots, \hat{B}_{i P}\right],
$$

with coefficients $\widehat{B}_{i p}$ corresponding to microphone at $\mathbf{r}_{p}$ and focal point at $\mathbf{r}_{i}$, and column vector $\mathbf{V}(\omega, l)$ is of the form

$$
\mathbf{V}=\left[\hat{V}_{1}(\omega, l), \hat{V}_{2}(\omega, l), \ldots, \hat{V}_{P}(\omega, l)\right]^{T} .
$$

For results presented in this paper, the steering vector coefficients $\widehat{B}_{i p}$ were constant for each focal point with a phase proportional to the distance between $\mathbf{r}_{p}$ and $\mathbf{r}_{i}$ and a magnitude inversely proportion to this distance. This weighting scheme resulted in good sidelobe behavior for all configurations used in collecting the experimental data.

The product pairs formed by the multiplication of the integrand in (4) result in $P^{2}$ products between all microphone signals, where $P$ of product pairs correspond to each microphone signal with itself, from which the individual microphone signal power is computed. Note that the correlations for the pairs of distinct microphones can be negative, depending the signal alignment. Since the power values for each individual microphone do not provide information related to the source location (i.e., signals will always be perfectly aligned independent of source positions), they can be subtracted out with no loss of spatial location information. The removal of this offset power is critical for the technique presented here, because at focal points without a source, a degree of symmetry exists between the positive and negative values. This behavior is exploited in a novel way to compute thresholds for sound source detection. While (4) explicitly shows computing the SRP value from all microphone signal products, it is more efficient to simply compute the power in the beamformed signal, as done in the typical SRP algorithm, and subtract the power of each individual microphone. This results in coherent power given by

$$
S_{C}\left(\mathbf{r}_{i}, l\right)=S\left(\mathbf{r}_{i}, l\right)-\sum_{p=1}^{P} \int_{\omega}\left|\hat{B}_{i p} \widehat{V}_{p}\left(\omega, \Delta_{l}\right)\right|^{2} d \omega .
$$

Coherent power values are computed on a set grid points in the FOV to form the pixels of SRCP image. The negative values of the SRCP image do not correspond to sources and therefore can be excluded when testing for potential targets; however, the distributions of the negative coherent power values are influenced by the power and position of noise sources, which makes these points useful in an adaptive thresholding scheme to maintain false-alarm rates. The accuracy of this scheme largely depends on the symmetry of the noise distribution at each pixel.

2.2. Expected Value of Noise Pixels. A symmetric distribution for $S_{c}$ in (7) implies an expected value of zero, as well as all odd order moments being zero. In this derivation, the expected value (first moment) is derived to identify the factors influencing deviations from 0 .

The vector multiplications of (4) result in $P^{2}$ terms, and the subtraction of autocorrelation terms in (7) effectively leave $P^{2}-P$ terms over which an expected value operator can be applied. The expected SRCP pixel value taken over all microphone pairs and FOV points becomes

$$
\mathrm{E}\left[S_{c}(l)\right]=\left(P^{2}-P\right) \int_{\omega} \mathrm{E}\left[\widehat{B}_{i p} \hat{B}_{i q}^{*} \widehat{V}_{p}(\omega, l) \hat{V}_{q}^{*}(\omega, l)\right] d \omega,
$$

for $p \neq q$. To identify the properties directly related to the microphone geometry, the complex elements of the steering vector are expressed in terms of the required scaling and time delay given by

$$
\widehat{B}_{i p}=B_{i p} \exp \left(j \omega \tau_{i p}\right) .
$$

For notational simplicity, assume that the $\beta$ of (3) is set to zero in order to substitute out $\widehat{V}_{p}(\omega, l)$ in the expected value of (8) with the expression in (2) and $\widehat{B}_{i p}$ with the expression of (9). Now assuming that distinct noise sources are uncorrelated, the expected value taken over all microphone pairs in the integrand of (8) takes on the form

$$
\begin{aligned}
\mathrm{E}\left[\widehat{B}_{i p} \widehat{B}_{i q}^{*} \hat{V}_{p}(\omega, l) \hat{V}_{q}^{*}(\omega, l)\right] \\
=\sum_{k=1}^{K} \mathrm{E}\left[\left|\hat{N}_{k}(\omega)\right|^{2}\right] \\
\quad \times \mathrm{E}\left[G_{k}(\omega) W_{i} \exp \left(j \omega\left(\left(\tau_{i p}-\tau_{k p}\right)-\left(\tau_{i q}-\tau_{k q}\right)\right)\right)\right],
\end{aligned}
$$

where $W_{i}=B_{i p} B_{i q}, G_{k}(\omega)=A_{k p}(\omega) A_{k q}^{*}(\omega)$.

The delays and weights associated with the microphone channels are typically not correlated with the noise source paths, which are reasonable when noise sources are sufficiently far from the point of interest in the FOV (typically outside of the main lobe of the beamfield). Therefore, they are assumed to be uncorrelated, so the microphone path terms can be factored out of the summation. Also, to investigate the statistics of the noise-only pixel relative to signal content and distribution geometry, the time delays 
are converted to spatial distances $d$, and frequencies to wavelengths $(\lambda)$ to rewrite the RHS of (10) as

$$
\begin{aligned}
& \mathrm{E}\left[W_{i} \exp \left(j 2 \pi\left(\frac{d_{i p}-d_{i q}}{\lambda}\right)\right)\right] \\
& \quad \times \sum_{k=1}^{K} \mathrm{E}\left[\left|\hat{N}_{k}(\omega)\right|^{2}\right] \mathrm{E}\left[G_{k}(\omega) \exp \left(j 2 \pi\left(\frac{d_{k q}-d_{k p}}{\lambda}\right)\right)\right] .
\end{aligned}
$$

Note that the exponential argument outside the summation is the microphone differential path length to the FOV point, and the exponential argument inside the summation is the noise differential path length to the FOV point.

The $W_{i}$ factors for each FOV point and microphone pair can be considered uncorrelated with the corresponding differential path length distances in the exponent outside the summation. This is a reasonable assumption, since these weights are typically not chosen based on the interpath distances to the FOV point. In addition, if the attenuations between effective noise sources and the microphones do not vary significantly over the room (compared to the differential noise path lengths to each FOV point), then these can be factored out of the exponent inside the summation to result in

$$
\begin{aligned}
& \bar{W}_{i} \mathrm{E}\left[\exp \left(j 2 \pi\left(\frac{d_{i p}-d_{i q}}{\lambda}\right)\right)\right] \\
& \quad \times \sum_{k=1}^{K} \mathrm{E}\left[\left|\hat{N}_{k}(\omega)\right|^{2}\right] \bar{G}_{k}(\omega) \mathrm{E}\left[\exp \left(j 2 \pi\left(\frac{d_{k q}-d_{k p}}{\lambda}\right)\right)\right],
\end{aligned}
$$

where $\bar{W}_{i}$ and $\bar{G}_{k}(\omega)$ are the mean values of $W_{i}$ and $G_{k}(\omega)$ over all microphone pairs and FOV points.

Equation (12) shows that the two complex exponential factors have the potential to drive the expected value to zero. The factor with the differential path lengths from the noise sources to the microphone pairs will be referred to as the noise-path factor. The other factor, due to the differential path lengths of the FOV point to microphone pairs, will be referred to as the mic-distribution factor. If the differential path lengths are on average much smaller than the source wavelengths, the phases are limited to a small range about zero, resulting in coherent sums at nonsource locations, which leads to noise coherence, distribution skewness, and false target identification. The coherent sums in this case relate to the spatial coherence length, in that changes in the FOV point location will result in changes in the differential path lengths. And if these changes are small relative to the wavelength, the coherent sum remains similar from one position to the next.

If the exponential argument is uniformly distributed from $-\pi$ to $\pi$ over all microphone pairs, the expected value of the complex exponential factor becomes zero. This condition will be especially important for the mic-distribution factor in (12), which scales all noise components. This factor is useful for a general analysis to determine performance, since it is based on the microphone distribution geometry, which is typically known or can be modified by the designer.

Let $\Delta_{p q}(i)$ be a random variable associated with the differential path lengths for location $\mathbf{r}_{i}$. It can be shown that for Gaussian distributed differential path lengths with standard deviation $\sigma_{\Delta}$ and mean zero, the expected value becomes

$$
\mathrm{E}\left[\exp \left(-j 2 \pi\left(\frac{\Delta_{p q}(i)}{\lambda}\right)\right)\right]=\exp \left(-2\left(\pi \frac{\sigma_{\Delta}}{\lambda}\right)^{2}\right),
$$

and for uniformly distributed differential path lengths, the expected value becomes

$$
\mathrm{E}\left[\exp \left(-j 2 \pi\left(\frac{\Delta_{p q}(i)}{\lambda}\right)\right)\right]=\operatorname{sinc}\left(\pi \frac{\sqrt{12} \sigma_{\Delta}}{\lambda}\right) .
$$

The relationships in (13) and (14) indicate that the expected value of the mic-distribution factor can never be identically zero over a range of frequencies, but it can be driven to increasingly smaller values by increasing $\sigma_{\Delta}$ relative to the source wavelengths. A zero-mean condition on the coherent power values is necessary for symmetry. However, the distribution can also be skewed from nonzero higherorder odd moments. Since higher-order moments result in more complicated relationships, only the impact on the expected value was derived here to see how well it predicts the impact on CFAR performance.

\section{Experimental Description and Analysis}

Equations (13) and (14) indicate that the mean value can be driven to small values by either high-pass filtering the source to diminish the impact of lower frequencies, or adjusting the microphone positions to increase the differential path length distribution over the FOV. To better understand the impact of these approaches to improve CFAR performance, experiments were designed to explore the relationships between distribution nonsymmetries, source spectral content, array geometry, and statistical models for threshold estimation.

3.1. Experimental Recordings. Figure 1 shows the three microphone distributions used. All geometries include 16 omnidirectional microphones (Behringer ECM8000) with the FOV being a $3 \mathrm{~m}$ by $3 \mathrm{~m}$ plane $1.57 \mathrm{~m}$ above the floor. The FOV plane was spatially sampled at $4 \mathrm{~cm}$ increments in the $X$ and $Y$ directions. Signals were amplified with Audio Buddy preamplifiers and sampled with two 8-channel Delta 1010 digitizers at $22.05 \mathrm{kHz}$ (both manufactured by M-Audio, Irwindal, CA) and downsampled to $16 \mathrm{kHz}$ for processing.

Figure 1(a) shows a schematic of the linear array placed 1.52 meters above the floor, $0.5 \mathrm{~m}$ away from the FOV edge. The linear microphone spacing was $0.23 \mathrm{~m}$ in this case. The array was symmetrically placed along the $y$-axis relative to the FOV. Figure 1(b) shows a perimeter array with microphones placed 1.52 meters above the floor, $0.5 \mathrm{~m}$ away from the FOV plane, and a microphone spacing of $0.85 \mathrm{~m}$ along the perimeter. Figure 1(c) shows the planar array with microphones placed in a plane $1.98 \mathrm{~m}$ above the ground in 


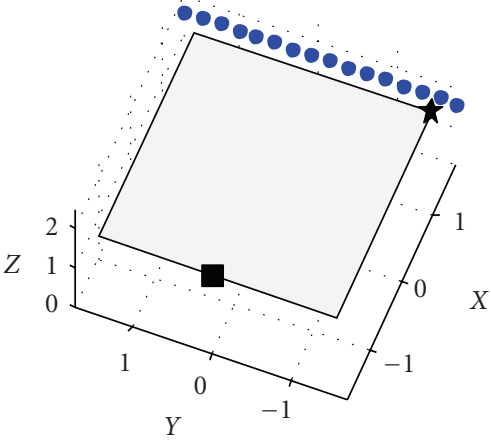

(a)

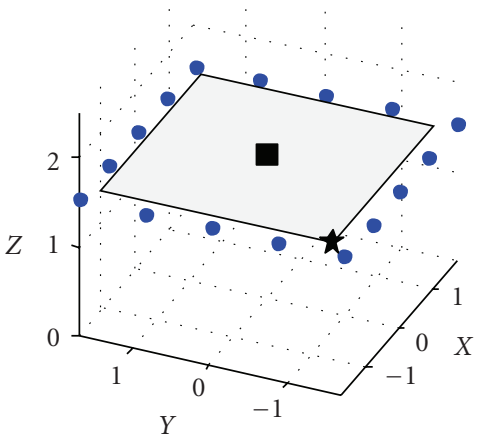

(b)

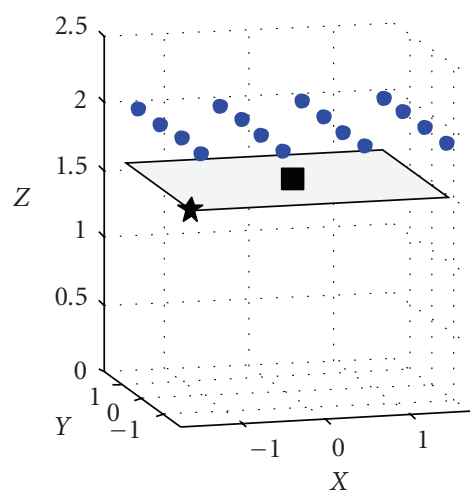

(c)

Figure 1: Microphone distributions and FOV (shaded plane) for simulation and experimental recordings with axes in meters. Small filled circles outside the FOV denote a microphone position, and the square and star markers in the FOV denote the smallest and largest (resp.) differential path distance standard deviation over all pairs: (a) linear, (b) perimeter, and (c) planar.

a rectangular grid starting on a corner directly above the FOV with a microphone spacing of $1 \mathrm{~m}$ in the $X$ and $Y$ directions.

Aluminum struts around the FOV held the microphones in place, and positions were measured manually multiple times with a laser meter and tape measure. Precision limits of the measurements were estimated to be within $\pm 2 \mathrm{~cm}$. Sound speeds were measured on the day of each recording, which was $347 \mathrm{~m} / \mathrm{s}$ for the linear array and $346 \mathrm{~m} / \mathrm{s}$ for the perimeter and planar arrays. Two speakers (Yamaha NS-E60 speakers) were paced outside the FOV approximately $2 \mathrm{~m}$ away from the FOV to act as white noise sources and create a nonstationary power distribution over the FOV. Relative to the geometries shown in Figure 1, the noise sources were placed beyond the negative $X$ and negative $Y$ axes.

Five separate recordings of 25 seconds each were made for the microphone geometries, and the white noise signals were varied for each recording. The SRCP images were created with the algorithm based on (7), where signals were partitioned into $20 \mathrm{~ms}$ segments $\left(\Delta_{l}\right)$ and incremented every $10 \mathrm{~ms}$ to create a sequence of the SRCP images. Scale values for the CFAR thresholds were estimated from the absolute values of negative pixels within a $15 \times 15$ neighborhood about the center (test) pixel. This resulted in a total of 46.5 million detection tests for estimating the FA probabilities. Various levels of high-pass filtering and partial whitening were applied before creating the SRCP images and testing CFAR performance. The level of partial whitening was controlled with the parameter $\beta$ in (3).

3.2. Differential Path Length Analysis. In order to determine the distributions of microphone differential path lengths, normalized histograms (compute from 240 microphone pairs for each FOV point) were plotted for two particular FOV positions corresponding to the maximum and minimum standard deviations. These positions are indicated with the square (minimum) and star (maximum) markers on the FOVs in Figure 1. Figure 2 shows the normalized histograms of the microphone differential path lengths and standard deviations for these points. Visual observation suggests the distributions are similar to Gaussian in that they have a central tendency, but they are also like the uniform distribution in their limited support. The uniform distribution results in a more conservative performance and represents a worse case, since the mean offset rolls off faster for the Gaussian assumption in (13) than that for the uniform assumption in (14). Therefore, the uniform distribution is used in the analyses to determine frequency limits for the acoustic sources based on array properties. Based on empirical observations, it was determined that frequencies larger than the third null of the sinc function (which are limited to $-20 \mathrm{~dB}$ or less from the maximum) typically result in good CFAR performance. Thus, highpass filtering the signal at this limit, or reducing their relative high-frequency contribution with the PHAT, reduces the low-frequency signal component contributions that the microphone distribution cannot properly decorrelate. Using the third null of the sinc function, the low-frequency limit can be computed from

$$
f_{L}=\frac{3 c}{\sigma_{\Delta} \sqrt{12}}
$$

where $c$ is the sound speed and $\sigma_{\Delta}$ is the standard deviation of the differential path lengths. For the linear, perimeter, and planar geometries, the lower frequency limits corresponding to the minimum standard deviations over the FOV are $1435 \mathrm{~Hz}, 790 \mathrm{~Hz}$, and $447 \mathrm{~Hz}$, respectively. These limits correspond to the worst-case position over the FOV. For a prediction of an average performance for the microphone geometry, the median of the standard deviations can be used. For the linear, perimeter, and planar geometries the median values are $.61,1.25$, and 1.13 respectively, and correspond to frequency limits of $493 \mathrm{~Hz}, 240 \mathrm{~Hz}$, and $266 \mathrm{~Hz}$. The impact of these limits on CFAR performance will be investigated in the next 2 sections. 


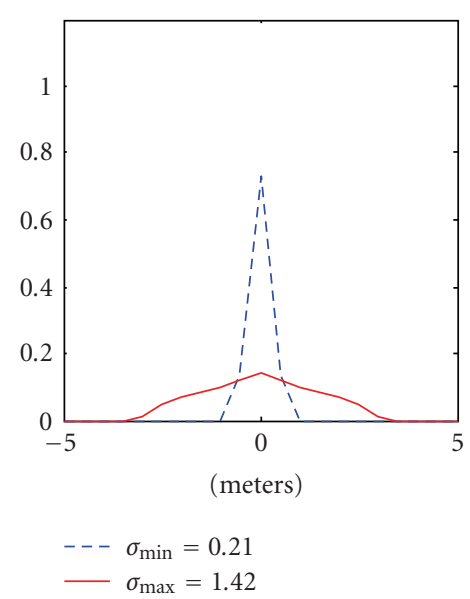

(a)

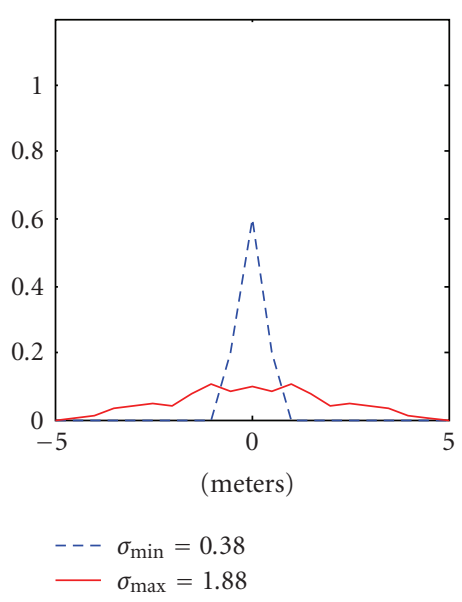

(b)

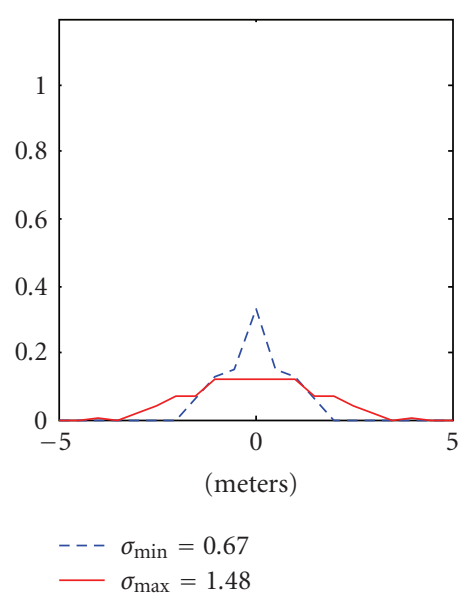

(c)

FIGURE 2: Normalized histograms for microphone pair differential path lengths at FOV points that generate the minimum and maximum standard deviations for (a) linear geometry, (b) perimeter geometry, and (c) planar geometry.

\section{Coherent Power Distribution Analysis}

This section examines the noise-only distributions for the positive and negative coherence values in a test neighborhood. Histograms were created by normalizing nonoverlapping $15 \times 15$ pixel neighborhoods by the root-mean square of the negative pixel values to reduce the effects of the nonstationary noise power over the SRCP images. Normalized coherent power values were binned over values ranging from 0 to 15 with 0.0125 intervals. The cumulative distribution functions (cdfs) were estimated from the normalized histograms, and the cdf complements (1-cdf) were plotted on a log scale to examine distribution tail differences between the positive and negative pixel absolute values. The complement cdf corresponds directly to the FA probability as a function of threshold.

Figure 3 compares the cdf complements of the positive and negative SRCP values for all geometries with two levels of high-pass filtering. The distances between the curves along the $x$-axis correspond to the error in the threshold estimation between the positive and negative pixels values. The relative deviations from symmetry, observed in Figure 3, are consistent with differential path length analyses of the previous section. The linear geometry exhibits the largest deviation from symmetry, while the perimeter and planar distributions are much less. A high-pass filter with cutoff frequency at $300 \mathrm{~Hz}$ was applied for the results shown in Figures 3(a), 3(c), and 3(e). For the planar and perimeter geometries, the cutoff frequency is higher than the lower limit required by (15) based on the median standard deviation $(266 \mathrm{~Hz}$ for planar and $240 \mathrm{~Hz}$ for perimeter), but the $300 \mathrm{~Hz}$ cutoff was less than the lower frequency limit for the linear geometry $(493 \mathrm{~Hz})$. Figures $3(\mathrm{~b}), 3(\mathrm{~d})$, and $3(\mathrm{f})$ show the corresponding results for a $1500 \mathrm{~Hz}$ high-pass filter cutoff which corresponds to frequencies greater than the minimum standard deviation for all geometries (for the linear geometry, this corresponded to $1435 \mathrm{~Hz}$ ). Minimal improvements result for the planar and perimeter geometries because $300 \mathrm{~Hz}$ was sufficient, while symmetry significantly improved for the linear geometry.

Figure 4 is analogous to Figure 3 with the addition of the PHAT (total whitening) being applied to the microphone channels. An overall improvement in symmetry is observed for all cases. The best symmetry is achieved for the perimeter array, with little improvement resulting from high-pass filtering at $1500 \mathrm{~Hz}$ (Figure 4(d)), since the highfrequency emphasis of the PHAT sufficiently reduced the impact of the lower frequencies. The linear geometry shows the most dramatic improvement as a result of high-pass filtering at $1500 \mathrm{~Hz}$ (Figures 4(a) and 4(b)) and the PHAT operation. Reasonable symmetry on the order of the other two geometries is achieved for the linear array in this case.

Finally, data were modeled with a Weibull distribution with cdf given by

$$
P\left(S_{c}\right)=1-\exp \left(\frac{S_{c}}{a}\right)^{b},
$$

where $a$ and $b$ are the scale and shape parameters, respectively. A maximum likelihood estimate of the Weibull parameters was performed on the SRCP image pixels (positive and negative values separately). These estimates provided an approximate range of shape parameters for the CFAR algorithm applied in the next section. Table 1 shows the shape parameter estimates for the two levels of filtering and three whitening levels. While total whitening results in the best distribution symmetry, previous work [11, 12, 16] showed that significantly better detection rates are achieved with partial whitening, rather than total whitening. Therefore, partial whitening results with $\beta=0.75$ are also included in the table.

\section{CFAR Performance Results and Discussion}

This section describes the CFAR threshold estimation and tests its performance. Based on the differences between 


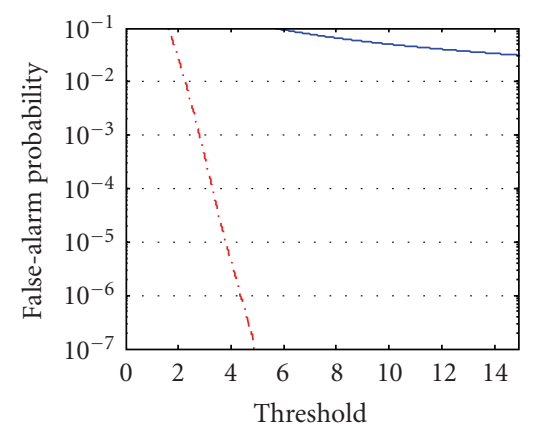

(a)

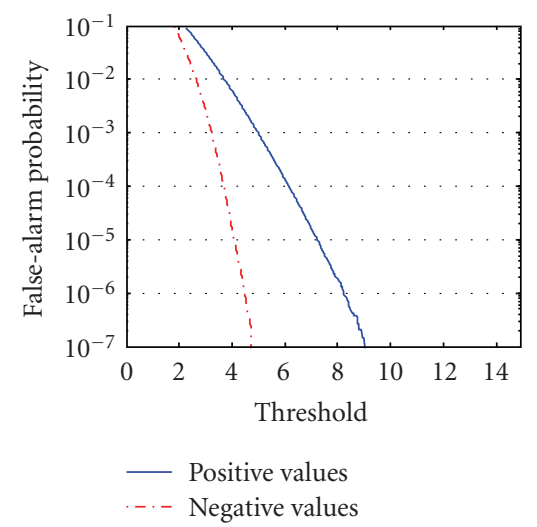

(d)

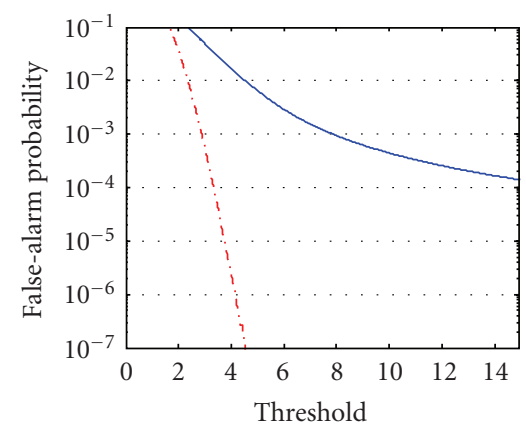

(b)

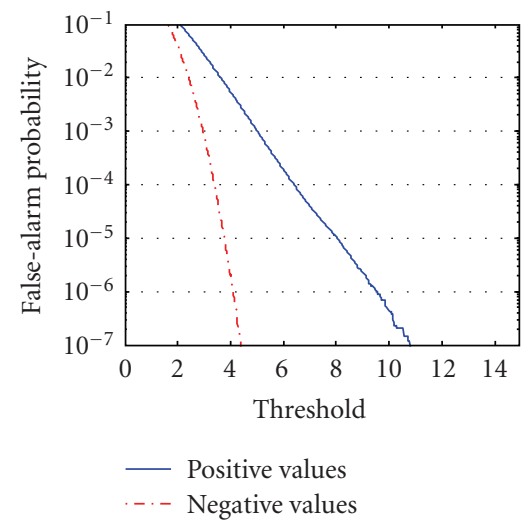

(e)

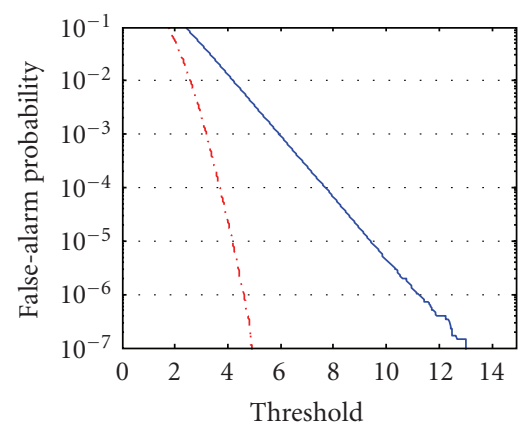

(c)

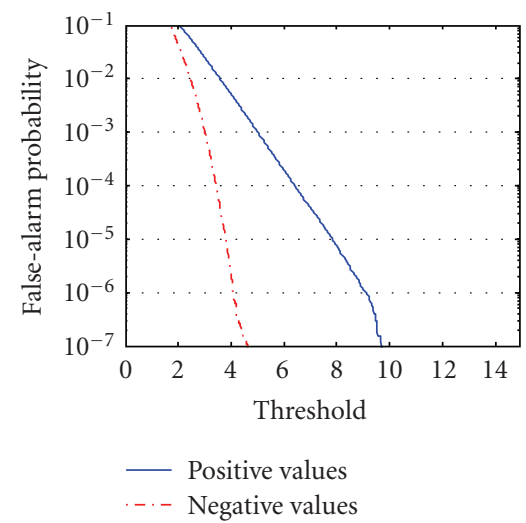

(f)

FIgURE 3: Cumulative distribution function complements for positive and negative SRCP values estimated from experimental data with high-pass filtering (a) linear array, $300 \mathrm{~Hz}$ cutoff (b) linear array, $1500 \mathrm{~Hz}$ cutoff (c) perimeter array, $300 \mathrm{~Hz}$ cutoff (d) perimeter array, $1500 \mathrm{~Hz}$ cutoff (e) planar array, and $300 \mathrm{~Hz}$ cutoff (f) planar array, $1500 \mathrm{~Hz}$ cutoff.

TABLE 1: Weibull parameter estimates for coherent power.

\begin{tabular}{|c|c|c|c|c|c|}
\hline \multirow{2}{*}{ Filter cutoff $(\mathrm{Hz})$} & \multirow{2}{*}{ Geometry } & \multirow{2}{*}{$\beta$} & \multicolumn{2}{|c|}{ Shape parameter $(b)$} & \multirow{2}{*}{$\%$ Difference } \\
\hline & & & Positive values & Negative values & \\
\hline \multirow{9}{*}{300} & \multirow{3}{*}{ Linear } & 0 & 0.52 & 1.69 & 106 \\
\hline & & 0.75 & 0.67 & 1.44 & 73 \\
\hline & & 1 & 0.98 & 1.36 & 33 \\
\hline & \multirow{3}{*}{ Perimeter } & 0 & 1.16 & 1.36 & 16 \\
\hline & & 0.75 & 1.19 & 1.30 & 9 \\
\hline & & 1 & 1.20 & 1.29 & 7 \\
\hline & \multirow{3}{*}{ Planar } & 0 & 1.17 & 1.36 & 15 \\
\hline & & 0.75 & 1.16 & 1.32 & 13 \\
\hline & & 1 & 1.17 & 1.32 & 12 \\
\hline \multirow{9}{*}{1500} & \multirow{3}{*}{ Linear } & 0 & 1.07 & 1.43 & 29 \\
\hline & & 0.75 & 1.16 & 1.33 & 14 \\
\hline & & 1 & 1.19 & 1.32 & 11 \\
\hline & \multirow{3}{*}{ Perimeter } & 0 & 1.18 & 1.36 & 14 \\
\hline & & 0.75 & 1.20 & 1.30 & 8 \\
\hline & & 1 & 1.21 & 1.29 & 7 \\
\hline & \multirow{3}{*}{ Planar } & 0 & 1.17 & 1.36 & 15 \\
\hline & & 0.75 & 1.17 & 1.31 & 11 \\
\hline & & 1 & 1.18 & 1.31 & 10 \\
\hline
\end{tabular}




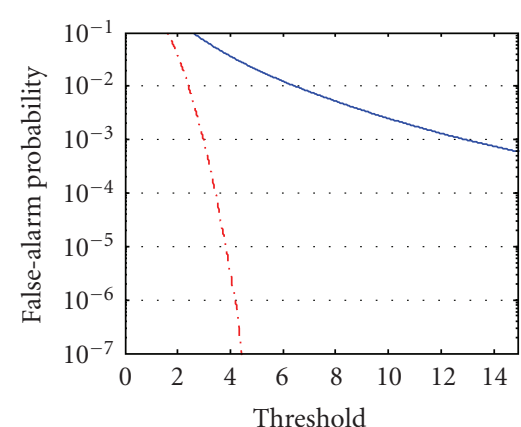

(a)

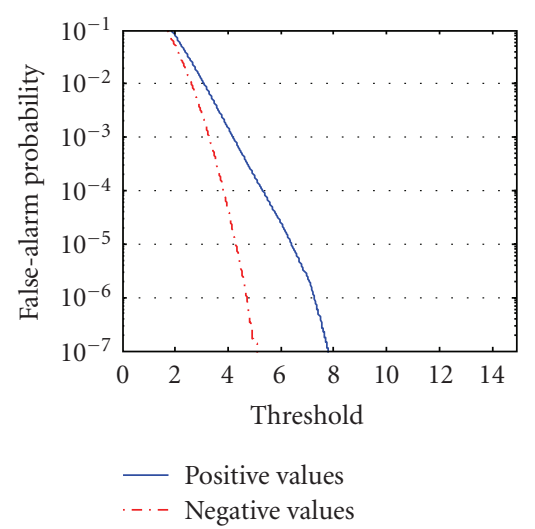

(d)

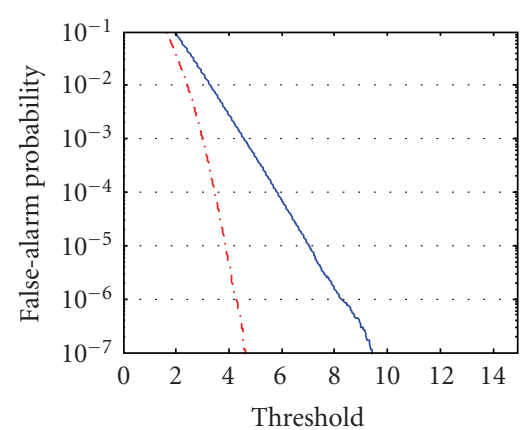

(b)

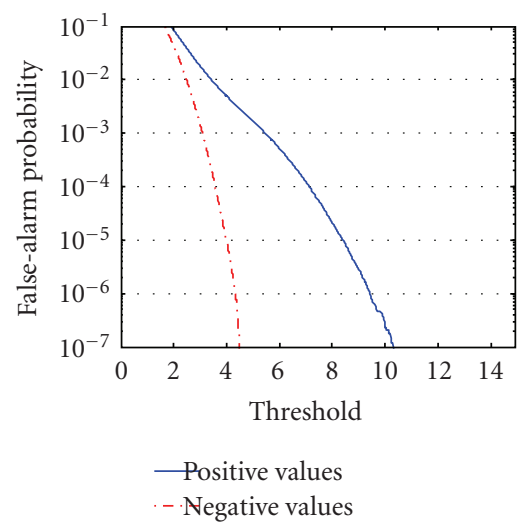

(e)

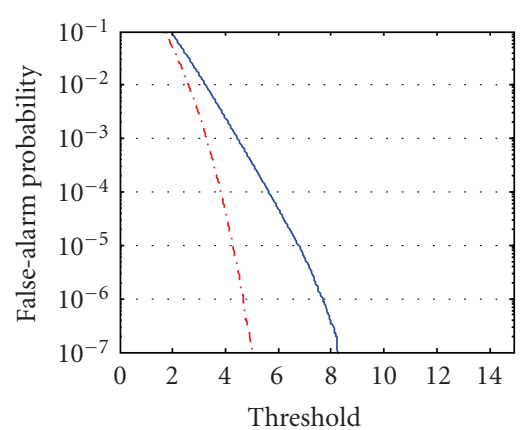

(c)

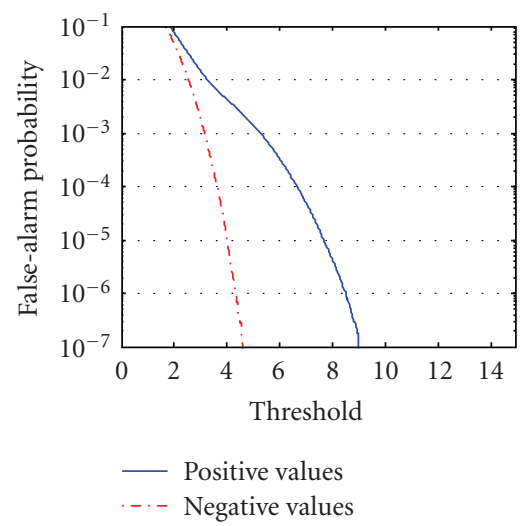

(f)

FIGURE 4: Cumulative distribution function complements for positive and negative SRCP values estimated from experimental data with high-pass filtering and whitening with the PHAT (a) linear array, $300 \mathrm{~Hz}$ cutoff (b) linear array, $1500 \mathrm{~Hz}$ cutoff (c) perimeter array, $300 \mathrm{~Hz}$ cutoff (d) perimeter array, $1500 \mathrm{~Hz}$ cutoff (e) planar array, and $300 \mathrm{~Hz}$ cutoff (f) planar array, $1500 \mathrm{~Hz}$ cutoff.

the distributions shown in the last section, a reasonable goal for good performance is to have FA probabilities remain within an order of magnitude of the desired FA probability over a broad range of desired FA probabilities $\left(10^{-6}\right.$ to $\left.10^{-1}\right)$.

5.1. CFAR Threshold Estimation and Results. The Weibull distribution was used primarily for its ability to model skewness via its shape parameter. The shape parameter, $b$, was selected based on the limited ranges shown in Table 1 . Therefore, given a known shape parameter, the scale parameter is computed from the negative coherent power values via maximum likelihood estimate

$$
\hat{a}=\left(\frac{1}{\left\|N_{0}^{-}\right\|} \sum_{S_{i} \in N_{0}^{-}}\left|S_{i}\right|^{b}\right)^{1 / b},
$$

where $S_{i}$ are the coherent powers in test pixel neighborhood set, $N_{0}$, with subset $N_{0}^{-}$denoting only the negative coherent power values, and $\left\|N_{0}^{-}\right\|$denotes the number of pixels in $N_{0}^{-}$. For a user specified FA probability, $P_{\mathrm{FA}}$, the test threshold is computed through the inverse compliment cdf of (16)

$$
\widehat{T}=\hat{a}\left[-\ln \left(P_{\mathrm{FA}}\right)\right]^{1 / b},
$$

where $P_{\mathrm{FA}}$ is the desired FA probability. The local-scale values for each test pixel are computed and substituted into (18) to compute the thresholds for each neighborhood. Experimental FA probabilities are computed as the number of times the test pixel value exceeds the threshold, divided by the total number of test points (46.4 million test points).

For the linear geometry, Figure 5 presents the ratio of experimental to desired FA probabilities versus the desired FA probabilities. The broken line on the plots is at a ratio of one, indicating an agreement between experimental and desired FA probabilities (target performance). Figure 5(a) shows differences larger than one order of magnitude between the desired and experimental FA probabilities for shape parameter $b=1.26$, and while some improvement is observed in Figure 5(b) as a result of selecting a lower $b$ (increased skewness), the best performance with cutoff frequency of $300 \mathrm{~Hz}$ corresponds to $b=0.6$. The ratios, however, still exceed an order of magnitude over the desired FA probability range. Thus, as the previous analysis predicted, the linear distribution has poor CFAR performance due to its limited differential microphone path differences.

To demonstrate the impact of the lower frequencies on this performance, the signals are high-pass filtered with a cutoff of $1500 \mathrm{~Hz}$. These results are presented in Figure 6. Note in Figure 6(a) that while the error is reduced over the cases shown in Figure 5, significant error still exists without whitening from the PHAT; however, with whitening, the FA probability ratios stay within one order of magnitude. 


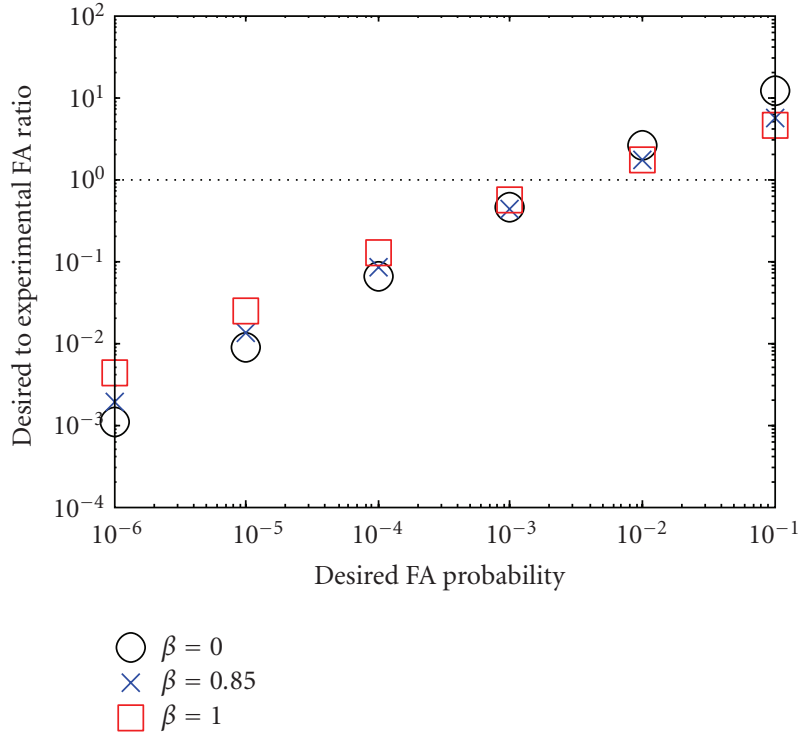

(a)

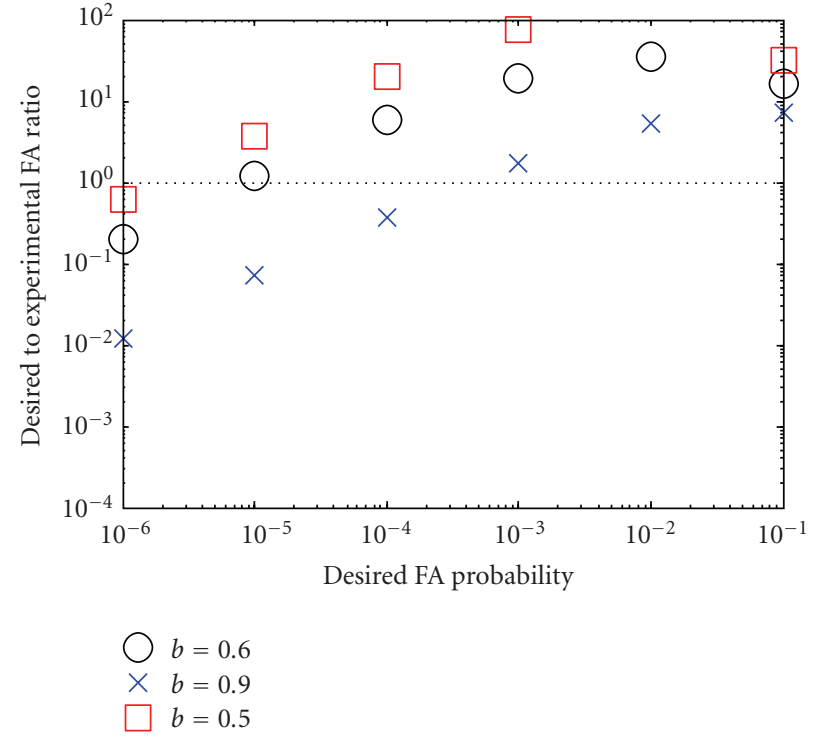

(b)

FIGURE 5: Ratios of specified to empirical (experimental) FA probabilities for linear array for high-pass filtered signals with cutoff frequency of $300 \mathrm{~Hz}$. (a) Variations of PHAT- $\beta$ parameters using shape parameter of 1.26 , (b) variations of shape parameters using beta equal to 0.85 .

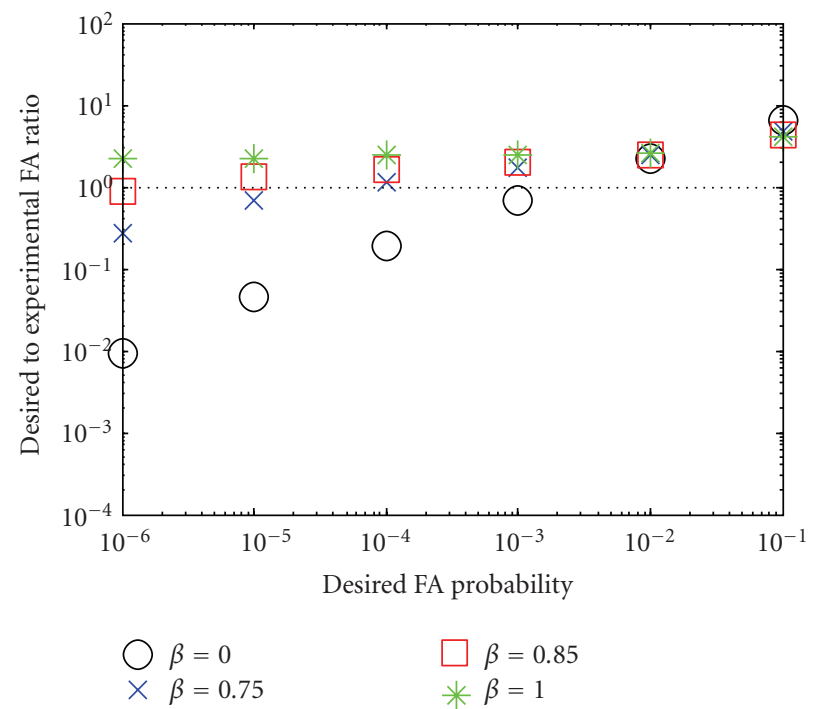

(a)

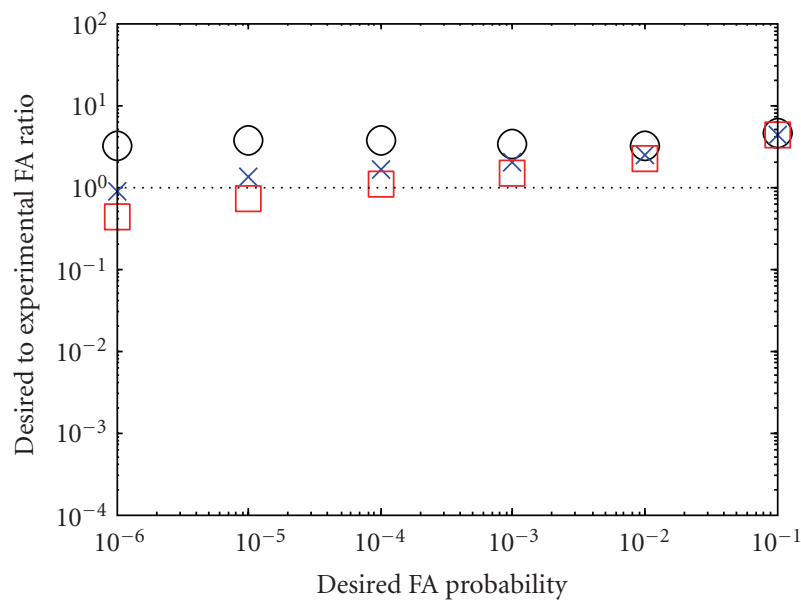

$$
\begin{aligned}
& \bigcirc b=1.2 \\
& \times b=1.26 \\
& \square b=1.3
\end{aligned}
$$

(b)

FIGURE 6: Ratios of specified to empirical (experimental) FA probabilities for linear array for high-pass filtered signals with cutoff frequency of $1500 \mathrm{~Hz}$. (a) Variations of PHAT- $\beta$ parameters using shape parameter of 1.26, (b) variations in shape parameters using beta equal to 0.85 .

Figure 6(b) demonstrates the performance sensitivity to the shape parameter, with the best performance achieved for shape parameter $b=1.26$ and good performance being maintained over the range from $b=1.2$ to 1.3 , which is consistent with the shape parameters shown in Table 1 for this case.

Figure 7 shows analogous results for the perimeter distribution. The previous analysis indicated lower frequency limits of $240 \mathrm{~Hz}$ and $790 \mathrm{~Hz}$ corresponding to the median and minimum standard deviations of the differential path lengths. While results high-pass filtered at $300 \mathrm{~Hz}$ satisfy over $50 \%$ of the pixels in the FOV, sufficient pixels existed requiring a higher cutoff frequency to impact the CFAR performance. Rather than increasing the cutoff as in the previous example, whitening was used to create a highfrequency emphasis to minimize the impact of these pixels. Note that Figure 7(a) shows that $b=1.26$ results in good CFAR performance provided a whitening operation is applied. Figure 7(b) shows a slight improvement when $b$ is increased to 1.3 . 


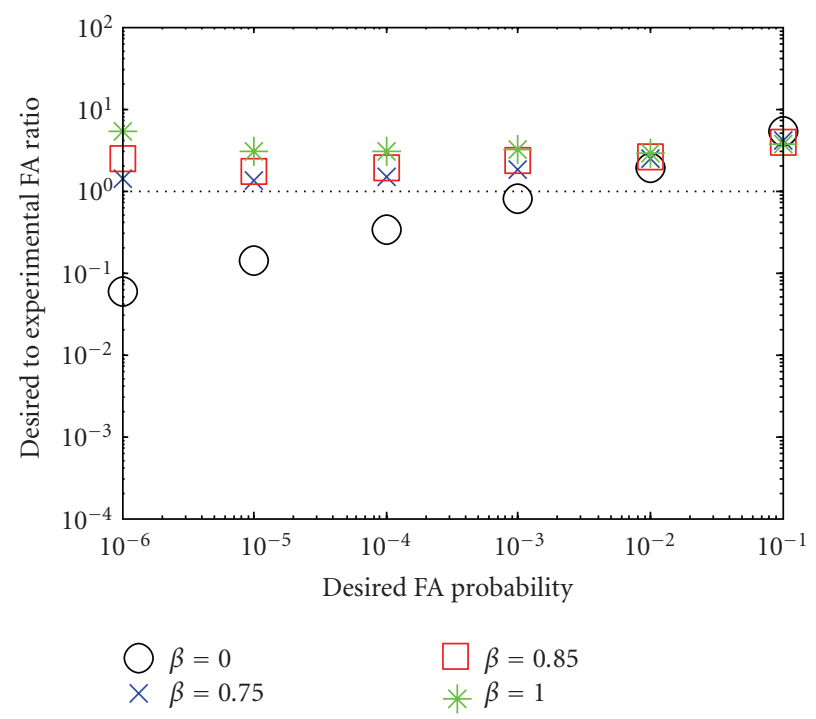

(a)

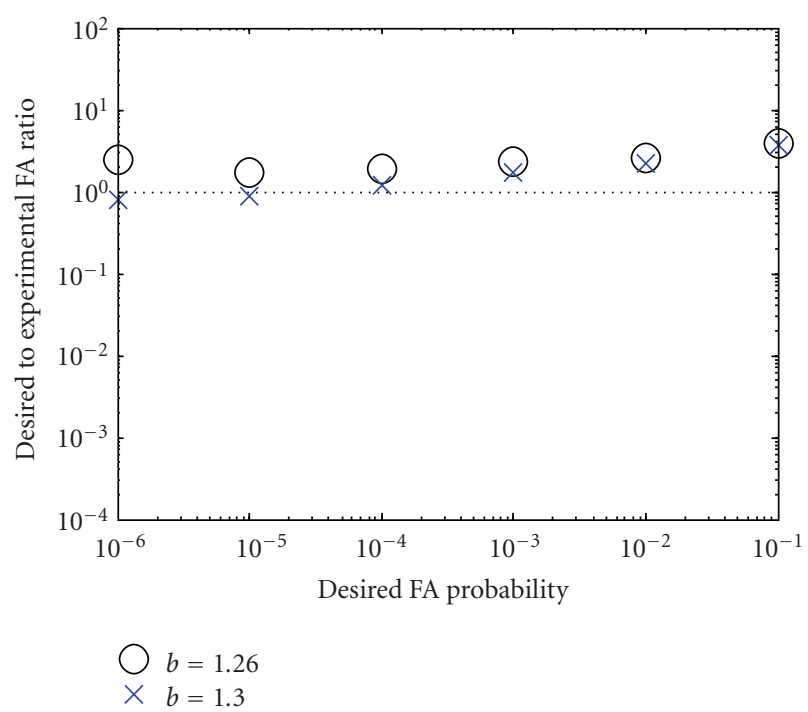

(b)

FIgURE 7: Ratios of specified to empirical (experimental) FA probabilities for perimeter array for high-pass filtered signals with cutoff frequency of $300 \mathrm{~Hz}$. (a) Variations in PHAT- $\beta$ parameters using shape parameter of 1.26, (b) variations in shape parameters using beta equal to 0.85 .

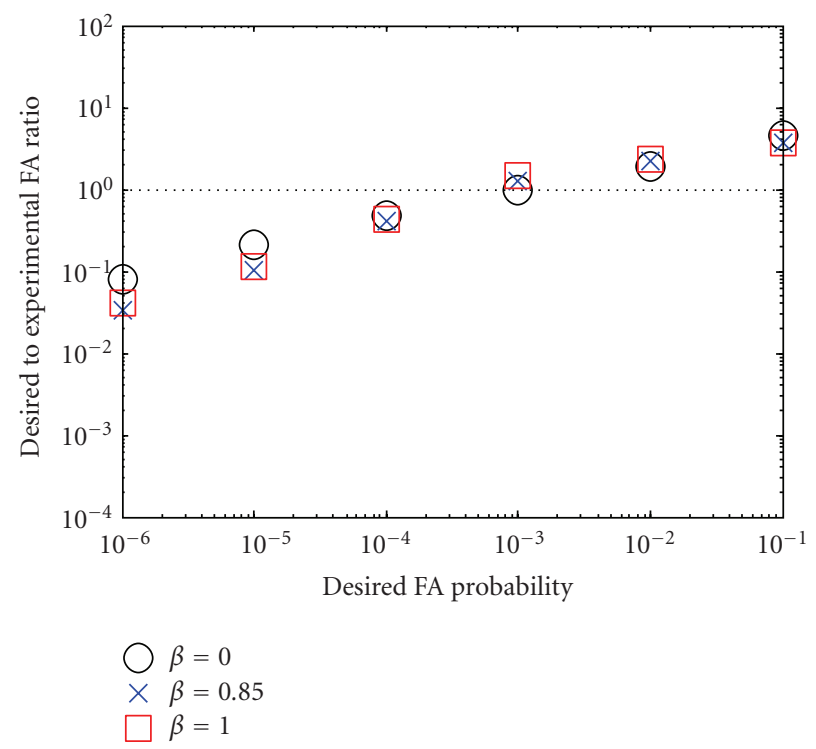

(a)

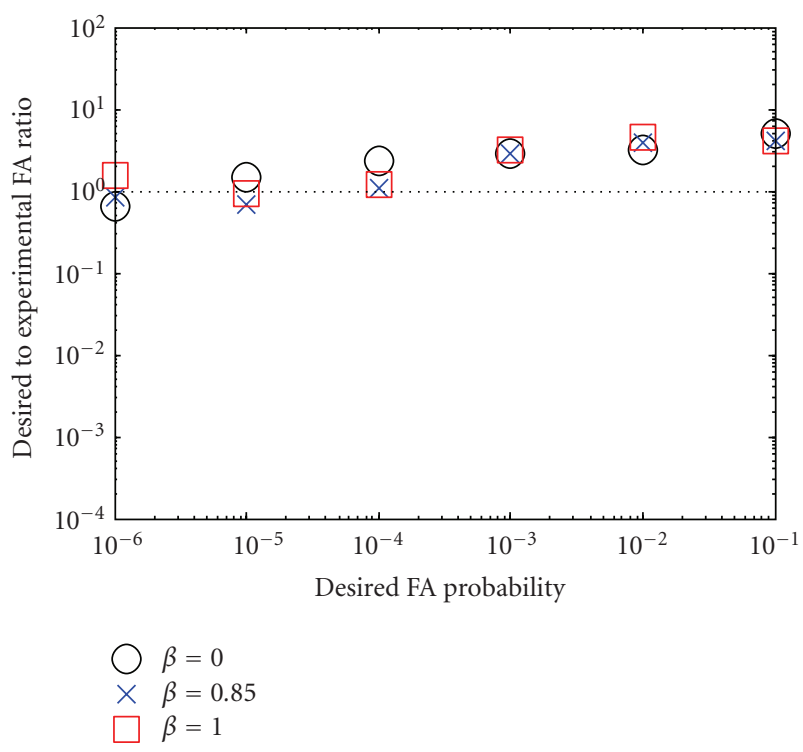

(b)

FIGURE 8: Ratios of specified to empirical (experimental) FA probabilities for planar array for high-pass filtered signals with cutoff frequency of $300 \mathrm{~Hz}$. (a) Variations in PHAT- $\beta$ parameters using shape parameter of 1.26 , (b) variations in PHAT- $\beta$ parameters, using shape parameter of 1.12 .

Results for the planar geometry are shown in Figure 8. In comparing Figures $7(\mathrm{a})$ and $8(\mathrm{a})$, the perimeter array shows superior CFAR performance, whereas whitening does not have an observable impact on CFAR performance for the planar distribution. The previous analysis showed a $266 \mathrm{~Hz}$ limit and a $447 \mathrm{~Hz}$ limit based on the median and minimum standard deviation, which is a more limited frequency range compared to the perimeter distribution, thus, explaining its performance being less sensitive to whitening. To improve performance, the high-pass filter can be set higher (i.e., to $500 \mathrm{~Hz}$ ), but this has practical disadvantages in that a significant amount of the signal power can exist below this cutoff. An alternative approach to compensate for the increased skewness is to decrease the Weibull shape parameter. Figure 8(b) shows the result of dropping $b$ to 1.12 , which is lower than the positive coherent 
power terms for this case shown in Table 1. While the error varies nonuniformly over the range tested, it remains within one order of magnitude.

5.2. Discussion of Results. Overall, results show that the perimeter array has the best performance in that it is least sensitive to lower frequencies. The high-pass filtering with a cutoff of $300 \mathrm{~Hz}$ and partial whitening result in improved performance over the whole FOV. In general, performance is improved for higher frequency sources; however, raising the high-pass filter cutoff frequency can reduce target detection sensitivity, so the other approaches are usually more desirable, such as whitening or adjusting the statistical models.

The linear and planar distributions did not perform as well as the perimeter distribution, as predicted by their differential path length standard deviations. In both cases, performance was improved by using a more skewed Weibull distribution to fit the data (Figures 5(b) and 8(b)). The increased distribution skewness compensates for some of the performance losses due to the nonsymmetries. In selecting a more skewed $b$ value for negative pixels, a larger-scale parameter estimate from (17) will result (for the same data). This bias increases the threshold, which compensates for the high levels of positively skewed values. This approach is limited in that if the shape parameters deviate too far from the actual data properties, consistent CFAR performance cannot be maintained over the range of desired FA probabilities. This was the case for the results shown in Figure 5.

Whitening is an important operation for reducing the noise distribution skewness as shown by comparing Figures 3 and 4 . Especially note that the distribution of the negative coherent power values does not change much as a result of whitening; however, there is a much larger reduction in skewness for the positive coherent power points. This partially explains why the PHAT improves SRP image appearance. The impulse/speckle noise resulting from the highly skewed noise pixels tends to create a distracting background from which to visually identify targets. The other advantage of whitening is that it reduces the correlation between adjacent pixels by emphasizing the higher frequencies. The increased spatial decorrelation or reduced correlation length for higher frequencies is indicated by the mic-distribution and noise-path factors of (12). Smaller wavelengths increase the sensitivity of the phase to changes in the differential path lengths as a result of spatial changes in the FOV. This not only improves noise distribution symmetry, but effectively increases the uncorrelated negative (noise) pixels in the test point neighborhood, which can reduce variations in the Weibull-scale parameter estimate.

For examples presented in this paper, a $15 \times 15$ pixel neighborhood was used. Other sizes also were examined (such as $7 \times 7$ ), and the $15 \times 15$ did the best as far as being the smallest neighborhood to achieve nearly the best performance for all three microphone arrays. One possible explanation for the poor performance of the linear array is that the neighborhood size was not large enough for good convergence of $\hat{a}$. Experimental results (not shown here) indicated that the linear array was more sensitive to the neighborhood size than the planar and perimeter distribution. A neighborhood of size $7 \times 7$ severely degrades the performance in the linear array. The CFAR performance for the planar and perimeter still remained within an order of magnitude for the $7 \times 7$ pixel neighborhood. However, increases in neighborhood size only resulted in incremental improvements for all arrays and eventual degradation due to the nonstationarity of the noise. So while the neighborhood size and limited correlation length of the linear array did contribute to its poor performance, the greater factor was the distribution skewness, as observed in Figures 3 and 4.

The standard deviations of the differential path lengths predicted the relative CFAR performance of the different microphone geometries. The frequency limits for each array as computed by (15) predicted the low-frequency limits with reasonable accuracy. For the linear array, however, these predictions were not as good. Acceptable performance for the linear distribution was not quite achieved by high-pass filtering at $1500 \mathrm{~Hz}$, which is greater than to the frequency required by its worst case FOV point $(1435 \mathrm{~Hz})$. Whitening was still required after this filtering for acceptable CFAR performance. This was in part due to not taking the noisepath factor into account.

The noise-path factor depends on the path lengths from the noise sources to the microphones and can vary as sources move in the environment. For this paper, however, the noise sources were stationary. For the linear array, one noise source was positioned broadside, nearly $5 \mathrm{~m}$ away. This resulted in a small differential path length variance and significantly reduced the decorrelation from noise-path factors in the summations. The perimeter and planar geometries had more endfire-like orientations to both major noise sources, thereby increasing the differential path variance for the noise-path factors and making it less of a factor in the performance. As a result, the shape parameters for fitting the Weibull distribution to the planar and perimeter coherent noise values were very close to the 1.26 (expected for Gaussian noise), whereas the linear geometry shape parameters deviated much more from the 1.26 level, even after high-pass filtering at $1500 \mathrm{~Hz}$.

\section{Conclusion}

This paper introduced a method for CFAR threshold estimation that uses the negative coherent power values in images created with SRP algorithms. Reasonable performance was obtained provided the source content was above the lower frequency limit associated with the array. An analysis based on differential path lengths was used to predict relative CFAR performance between microphone distribution geometries based on the source frequency limit. It was shown that good CFAR performance could be obtained for microphone arrays with large differential path length variations over all microphone pair combinations relative to the signal source wavelengths. The analysis requires a standard deviation computation of the differential path lengths between microphone pairs and FOV points, which can be done for any 
geometry and is especially useful for systems with irregularly positioned microphones and FOV regions.

\section{Acknowledgment}

This work was supported in part by the National Science Foundation EPSCoR Program (Award 0447479).

\section{References}

[1] J. L. Flanagan, D. A. Berkley, G. W. Elko, J. E. West, and M. M. Shondhi, "Autodirective microphone systems," Acoustica, vol. 73, pp. 58-71, 1991.

[2] F. Khalil, J. P. Jullien, and A. Gilloire, "Microphone array for sound pickup in teleconference systems," AES: Journal of the Audio Engineering Society, vol. 42, no. 9, pp. 691-700, 1994.

[3] C. Che, M. Rahim, and J. Flanagan, "Robust speech recognition in a multimedia teleconferencing environment," Journal of the Acoustical Society of America, vol. 92, no. 4, p. 2476, 1992.

[4] D. Giuliani, M. Omologo, and P. Svaizer, "Talker localization and speech recognition using a microphone array and a crosspower spectrum phase analysis," in Proceedings of the International Conference on Spoken Language Processing (ICSLP '94), vol. 3, pp. 1243-1246, September 1994.

[5] T. B. Hughes, H. S. Kim, J. H. Dibiase, and H. F. Silverman, "Performance of an HMM speech recognizer using a real-time tracking microphone array as input," IEEE Transactions on Speech and Audio Processing, vol. 7, no. 3, pp. 346-349, 1999.

[6] H. F. Silverman, "Some analysis of microphone arrays for speech data acquisition," IEEE Transactions on Acoustics, Speech, and Signal Processing, vol. 35, no. 12, pp. 1699-1712, 1987.

[7] S. M. Yoon and S. C. Kee, "Speaker detection and tracking at mobile robot platform," in Proceedings of the International Symposium on Intelligent Signal Processing and Communication Systems (ISPACS '04), pp. 596-600, November 2004.

[8] T. S. Huang, "Multimedia/multimodal signal processing, analysis, and understanding," in Proceedings of the 1st International Symposium on Control, Communications and Signal Processing, p. 1, 2004.

[9] J. H. DiBiase, H. F. Silverman, and M. S. Brandstein, "Robust localization in reverberant rooms," in Microphone Arrays, Signal Processing Techniques and Applications, pp. 157-180, Springer, New York, NY, USA, 2001.

[10] T. Gustafsson, B. D. Rao, and M. Trivedi, "Source localization in reverberant environments: modeling and statistical analysis," IEEE Transactions on Speech and Audio Processing, vol. 11, no. 6, pp. 791-803, 2003.

[11] K. D. Donohue, J. Hannemann, and H. G. Dietz, "Performance of phase transform for detecting sound sources with microphone arrays in reverberant and noisy environments," Signal Processing, vol. 87, no. 7, pp. 1677-1691, 2007.

[12] A. Ramamurthy, H. Unnikrishnan, and K. D. Donohue, "Experimental performance analysis of sound source detection with SRP PHAT- $\beta$," in Proceedings of the IEEE Southeastcon, pp. 422-427, March 2009.

[13] H. Rohling, "Radar CFAR thresholding in clutter and multiple target situations," IEEE Transactions on Aerospace and Electronic Systems, vol. 19, no. 4, pp. 608-621, 1983.

[14] K. D. Donohue and N. M. Bilgutay, "OS characterization for local CFAR detection," IEEE Transactions on Systems, Man and Cybernetics, vol. 21, no. 5, pp. 1212-1216, 1991.
[15] S. Kuttikkad and R. Chellappa, "on-Gaussian CFAR techniques for target detection in highresolution SAR images, image processing," in Proceedings of the IEEE International Conference on Image Processing (ICIP'94), vol. 1, pp. 910-914, November 1994.

[16] K. D. Donohue, K. S. McReynolds, and A. Ramamurthy, "Sound source detection threshold estimation using negative coherent power," in Proceedings of the SouthEast Conference, pp. 575-580, April 2008. 